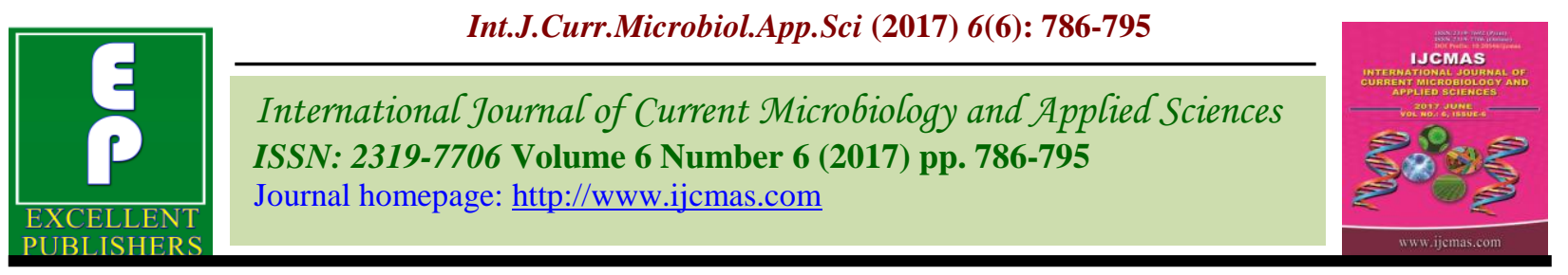

Original Research Article

https://doi.org/10.20546/ijcmas.2017.606.092

\title{
Character Association and Path Analysis in Cowpea [Vigna unguiculata (L.) Walp] Germplasm Line
}

\author{
Mahesh Sharma*, P.P. Sharma, B. Upadhyay, H.L. Bairwa and D.R. Meghawal \\ Department of Plant Breeding and Genetics Rajasthan College of Agriculture, Udaipur, India \\ *Corresponding author
}

A B S T R A C T

The present experiment was conducted using 60 genotypes of cowpea to study their correlation and path analysis during kharif-2015. It can be concluded from these

\begin{tabular}{|c|}
\hline Keywords \\
\hline $\begin{array}{l}\text { Correlation, } \\
\text { Flowering, } \\
\text { Analysis, } \\
\text { Possibilities. }\end{array}$ \\
\hline Article Info \\
\hline $\begin{array}{l}\text { Accepted: } \\
\text { 14 May } 2017 \\
\text { Available Online: } \\
\text { 10 June } 2017\end{array}$ \\
\hline
\end{tabular}
experiment findings that main yield contributing traits are biological yield per plant, number of pods per plant, number of flowers per plant, test weight, number of pods per cluster, pod length, number of seeds per pod, number of clusters per plant, harvest index and plant height due to their direct high positive association with seed yield. The trait days to maturity had negative and non-significant correlation with seed yield per plant thereby indicating selection for early maturity would give drought tolerant and drought avoiding genotypes affecting the seed yield positively in cowpea. Path analysis revealed that, seed yield per plant can be improved practicing selection for biological yield per plant, harvest index, number of pods per plant, days to $50 \%$ flowering, number of flowers per cluster, number of primary branches per plant, number of seeds per pod, test weight and plant height as they contributed directly to the seed yield per plant as revealed from path analysis. It indicated the possibilities of simultaneous improvement of these traits by selection. This in turn, will improve the seed yield, since they are positively correlated with the seed yield.

\section{Introduction}

Cowpea (Vigna unguiculata L. Walp) is a diploid species with $2 \mathrm{n}=2 \mathrm{x}=22$ chromosomes. It is a self-pollinated crop, with natural crosspollination of up to one percent. Cowpea belongs to the class of Dicotyledonea, order Fabales, family Fabaceae, subfamily Faboideae, tribe Phaseoleae, subtribe Phaseolinae, and genus Vigna (Pasquet et al., 2001). The primary gene-pool is composed of the domesticated cowpea ( $V$. unguiculata subsp. unguiculata var. unguiculata) and its wild progenitor (V. unguiculata subsp. unguiculata var. spontanea. The secondary gene-pool of cowpea includes nine perennial subspecies (Mebeaselassie et al., 2011). All cultivated cowpeas are grouped under the species Vigna unguiculata, which is subdivided into four cultivars group such as unguiculata (common cowpea used as food and fodder), sesquipedalis (the yard-long or asparagus bean used as vegetables), biflora (catjang) and textilis (used for fibers). The cultivar group of unguiculata is the most diverse of the four and is widely grown in Africa, Asia and Latin America. Arthur et al., (2009) mentioned that cowpea is the second most important pulse crop after groundnut, cultivated in Africa. Correlation analysis is an 
easy to use technique which provides information that selection for one character results in progress for other positively correlated characters. The importance of correlation studies in selection programmes is appreciable when highly heritable characters are associated with the important character like yield. Path coefficient is an excellent means of studying direct and indirect effects of interrelated components of a complex trait particularly if the high correlation between two traits is a consequence of the indirect effect of other traits (Bizeti et al., 2004). Pathcoefficient analysis measures the direct influence of one variable on another. By determining the inter-relationships among grain yield components, a better understanding of both the direct and indirect effects of the specific components can be attained (Chaudhary et al., 2005).

\section{Materials and Methods}

\section{Experimental site and materials}

The present investigation was carried out during Kharif 2015-16 at the Research Farm of Plant Breeding and Genetics, Rajasthan college of Agriculture, MPUAT, Udaipur. This experiment material comprised of sixty diverse genotypes including three checks viz.,RC-101, RC-19 and RCV-7 of cowpea. The experimental material of cowpea were sown in randomized block design in three replications. Two rows of each genotype were sown in a plot of $4 \mathrm{~m}$ length. The row to row and plant to plant distance were kept at $30 \mathrm{~cm}$ and $10 \mathrm{~cm}$, respectively. All the recommended package of practices were be followed to raise a healthy crop.

\section{Data collection and analysis}

The observations were recorded for 16 characters viz, Days to $50 \%$ flowering, Number of flowers per plant, Number of flowers per cluster, Days to maturity, Plant height, Number of primary branches per plant, Number of pods per plant, Number of clusters per plant, Number of pods per cluster, Pod length, Number of seeds per pod, Test weight, Seed yield per plant, Biological yield per plant, Harvest index and Seed protein content on five randomly selected plants from each genotypes in all the replications while days to $50 \%$ flowering and days to maturity which were recorded on plot basis. The phenotypic and genotypic correlation coefficients of all the characters were worked out as per the procedure suggested by Fisher (1954) and Al-Jibouri et al., (1958) and the path coefficient analysis was carried out as per the method suggested by Dewey and Lu (1959) at both phenotypic and genotypic level.

\section{Results and Discussion}

\section{Correlation coefficient}

Estimates of correlation coefficient at phenotypic and genotypic level are given in table 1. seed yield per plant exhibited significant positive correlation with biological yield $(0.739 * *)$, number of pods per plant $(0.453 * *)$, number of flowers per plant $\left(0.429^{* *}\right)$, test weight $\left(0.421^{* *}\right)$, number of pods per cluster $\left(0.373^{* *}\right)$, pod length $(0.351 * *)$, number of seeds per pod $(0.343 * *)$, number of clusters per plant $\left(0.318^{* *}\right)$, harvest index $\left(0.307^{* *}\right)$ and plant height $(0.252 * *)$, respectively at genotypic level. Biological yield per plant $(0.718 * *)$ followed by number of pods per plant $\left(0.419^{* *}\right)$, test weight $\left(0.410^{* *}\right)$, number of flowers per plant $\left(0.383^{* *}\right)$, harvest index $(0.340 * *)$, number of seeds per pod $\left(0.325^{* *}\right)$, pod length $\left(0.319^{* *}\right)$, number of clusters per plant $(0.272 * *)$, plant height $(0.248 * *)$ and number of pods per cluster $(0.189 *)$ showed positive highly significant correlation with seed yield per plant, respectively at phenotypic level. The present findings are in accordance with the findings 
of Leeliji et al., (1981), Padi et al., (2003), Fana et al., (2004), Kaveris et al., (2007) and Manggoel et al., (2012).

Seed protein content showed negative significant correlation with days to maturity (rg -0.198** and rp -0.187*). However, harvest index also showed highly significant and negative correlation with biological yield per plant (rg - $0.396^{* *}$ and $\mathrm{rp}-0.375^{* *}$ ) and days to maturity also showed significant and negative correlation with harvest index (rg $0.232 * *$ and $\mathrm{rp}-0.191 *)$. The present results are also finds out by Fikru et al., (2004) and Kaveris et al., (2007). Biological yield per plant exhibited highly significant and positive correlation with test weight $\left(\operatorname{rg} 0.398^{* *}\right.$ and rp $0.397 * *$ ), number of seeds per pod (rg $0.386 * *$ and $\mathrm{rp} 0.369^{* *}$ ), pod length (rg $0.353^{* *}$ and $\left.\mathrm{rp} 0.314^{* *}\right)$, and biological yield per plant showed negative significant correlation with days to $50 \%$ flowering (rg $0.189 *$ ) by Leleji (1981), Uguru (1996) and Manggoel et al., (2012). Test weight exhibited significant and positive correlation with pod length $(\operatorname{rg} 0.602 * *$ and $\operatorname{rp~} 0.527 * *)$, number of primary branches per plant (rg $0.259^{* *}$ and $\mathrm{rp} 0.255^{* *}$ ) However, test weight also showed significant and negative correlation with number of pods per plant (rg $-0.165^{*}$ and $\left.-\mathrm{rp} 0.149^{*}\right)$ by Fana et al., (2004), Fikru (2004) and Kaveris et al., (2007). Number of seeds per pod showed significant and positive correlation with pod length ( $\mathrm{rg} 0.366^{* *}$ and $\mathrm{rp} 0.401^{* *}$ ), number of primary branches per plant ( $\operatorname{rg~} 0.217^{* *}$ and rp 0.206**) and plant height ( $r g$ 0.160* and rp $0.153^{*}$ ) However, number of seeds per pod also showed significant and negative correlation with days to $50 \%$ flowering (rg $0.496 * *$ and $\mathrm{rp}-0.272 * *)$ and days to maturity ( $\mathrm{rg}-0.273^{* *}$ and $\mathrm{rp}-0.231 * *$ ) by Padi (2003) and Diriba Shanko et al., (2014). Pod length showed significant positive correlation with number of flowers per cluster

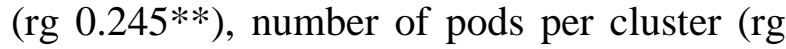
$0.219 * *)$ and number of primary branches per plant (rg 0.189* and rp 0.157*) Pod length also showed significant and negative correlation with days to $50 \%$ flowering (rg $0.426^{*}$ and $\mathrm{rp}-0.150^{*}$ ) and days to maturity (rg -0.236* and rp -0.167*) by Manggoel et al., (2012) and Diriba Shanko et al., (2014). Pods per cluster was exhibited highly significant and positive correlation with number of flowers per clusters (rg $0.823 * *$ and rp $0.637^{* *}$ ), number of flowers per plant (rg 0.494** and rp 0.250**) However, it was exhibited significant and negative correlation with number of clusters per plant (rp $0.374 * *)$ and days to $50 \%$ flowering (rg $0.178 *$ ) by Veeraswamy et al., (1973) and Vange et al., (2009). Pods per plant was exhibited highly significant and positive correlation with number of flowers per plant ( rg 0.933** and rp 0.822**). However, it was also exhibited significant and negative correlation with number of flowers per cluster ( $\left.\mathrm{rg}-0.245^{* *}\right)$ by Venkatesan et al., (2003) and Diriba Shanko et al., (2014). It can be concluded from these experiment findings that main yield contributing traits are biological yield per plant, number of pods per plant, number of flowers per plant, test weight, number of pods per cluster, pod length, number of seeds per pod, number of clusters per plant, harvest index and plant height due to their direct high positive association with seed yield. It indicated the possibilities of simultaneous improvement of these traits by selection. This in turn, will improve the seed yield, since they are positively correlated with the seed yield.

\section{Path coefficient analysis}

The direct and indirect effects of fifteen dependent characters on seed yield per plant as independent character was obtained in path coefficient analysis using genotypic correlation coefficient are presented in table 2. The highest positive direct effect on seed yield per plant was exhibited by biological 
Table.1 Genotypic and phenotypic correlation (*and ** significance levels of 5\% and $1 \%$ respectively)

\begin{tabular}{|c|c|c|c|c|c|c|c|c|c|c|c|c|c|c|c|c|c|c|}
\hline No & \multicolumn{2}{|l|}{ Character } & $\begin{array}{c}\text { Days to 50\% } \\
\text { flowering }\end{array}$ & $\begin{array}{l}\text { Number of } \\
\text { flowers/ plant }\end{array}$ & $\begin{array}{c}\begin{array}{c}\text { Number } \\
\text { of } \\
\text { flowers/ } \\
\text { cluster }\end{array} \\
\end{array}$ & $\begin{array}{c}\text { Days to } \\
\text { maturity }\end{array}$ & $\begin{array}{c}\text { Plant } \\
\text { height } \\
(\mathbf{c m})\end{array}$ & $\begin{array}{c}\text { Number } \\
\text { of } \\
\text { primary } \\
\text { branches/ } \\
\text { plant }\end{array}$ & $\begin{array}{c}\text { Number } \\
\text { of pods/ } \\
\text { plant }\end{array}$ & $\begin{array}{c}\begin{array}{c}\text { Number } \\
\text { of } \\
\text { clusters/ } \\
\text { plant }\end{array} \\
\end{array}$ & $\begin{array}{l}\text { Number } \\
\text { of pods/ } \\
\text { cluster }\end{array}$ & $\begin{array}{c}\text { Pod } \\
\text { length } \\
\text { (cm) }\end{array}$ & $\begin{array}{c}\text { Number } \\
\text { of seeds/ } \\
\text { pod }\end{array}$ & $\begin{array}{c}\text { Test } \\
\text { weight } \\
\text { (g) }\end{array}$ & $\begin{array}{l}\text { Biological } \\
\text { yield/ } \\
\text { plant }(g)\end{array}$ & $\begin{array}{l}\text { Harvest } \\
\text { index \% }\end{array}$ & $\begin{array}{c}\text { Seed } \\
\text { protein } \\
\text { content } \\
\% \\
\end{array}$ & $\begin{array}{c}\text { Seed } \\
\text { yield/ } \\
\text { plant } \\
(\mathrm{g} .)\end{array}$ \\
\hline \multirow[t]{2}{*}{1} & \multirow{2}{*}{$\begin{array}{l}\text { Days to } 50 \% \\
\text { flowering }\end{array}$} & $P$ & 1.000 & -0.026 & $-0.203^{* *}$ & $0.578^{* *}$ & 0.066 & -0.060 & 0.066 & 0.133 & -0.088 & $-0.150^{*}$ & $-0.272^{* * *}$ & -0.081 & -0.130 & 0.045 & -0.070 & -0.094 \\
\hline & & $\mathrm{G}$ & 1.000 & -0.076 & $-0.238^{* * *}$ & $0.790 * *$ & 0.080 & $\begin{array}{c}-0.100 \\
\end{array}$ & 0.046 & 0.136 & $-0.178^{*}$ & $-0.426 * *$ & $-0.496^{* *}$ & $\begin{array}{l}-0.106 \\
\end{array}$ & $-0.189^{*}$ & 0.017 & $\begin{array}{c}-0.090 \\
\end{array}$ & $-0.180 *$ \\
\hline \multirow[t]{2}{*}{2} & \multirow{2}{*}{$\begin{array}{l}\text { Number of flowers/ } \\
\text { plant }\end{array}$} & $P$ & & 1.000 & 0.136 & -0.084 & 0.121 & -0.030 & $0.822 * *$ & $0.641^{* *}$ & $0.250^{* *}$ & -0.068 & 0.051 & -0.118 & $0.285^{* *}$ & 0.121 & 0.033 & $0.383^{* * *}$ \\
\hline & & $\mathrm{G}$ & & 1.000 & 0.089 & -0.112 & 0.137 & -0.065 & $0.933^{* * *}$ & 0.791 ** & $0.494 * *$ & -0.0881 & 0.049 & -0.133 & $0.321 * *$ & 0.114 & 0.031 & $0.429 * *$ \\
\hline \multirow[t]{2}{*}{3} & \multirow{2}{*}{$\begin{array}{l}\text { Number of } \\
\text { flowers/cluster }\end{array}$} & $P$ & & & 1.000 & -0.042 & -0.007 & 0.132 & -0.123 & $-0.568^{* * *}$ & $0.637^{* * *}$ & 0.054 & -0.004 & 0.023 & 0.044 & -0.029 & -0.006 & 0.012 \\
\hline & & G & & & 1.000 & -0.071 & -0.005 & $0.199 * *$ & $-0.245^{* * *}$ & $-0.603^{* *}$ & $0.823 * *$ & $0.245 * *$ & 0.061 & 0.039 & 0.108 & -0.124 & -0.028 & 0.019 \\
\hline \multirow[t]{2}{*}{4} & \multirow{2}{*}{ Days to maturity } & $\mathrm{P}$ & & & & 1.000 & 0.135 & 0.137 & $\begin{array}{l}-0.054 \\
\end{array}$ & $\begin{array}{ll}-0.008 \\
\end{array}$ & -0.063 & $-0.167^{*}$ & -0.231 ** & 0.062 & 0.101 & $-0.191 *$ & $-0.187 *$ & \begin{tabular}{ll|}
-0.061 \\
\end{tabular} \\
\hline & & $\mathrm{G}$ & & & & 1.000 & 0.140 & $0.144 *$ & $\begin{array}{l}-0.062 \\
\end{array}$ & -0.030 & -0.089 & $-0.236^{* * *}$ & $-0.273^{* *}$ & 0.065 & 0.104 & $-0.232^{* * *}$ & $-0.198^{* *}$ & $\begin{array}{l}-0.074 \\
\end{array}$ \\
\hline \multirow[t]{2}{*}{5} & \multirow{2}{*}{ Plant height $(\mathrm{cm})$} & $P$ & & & & & 1.000 & 0.058 & 0.128 & 0.089 & 0.018 & -0.101 & $0.153^{*}$ & 0.012 & $0.300 * *$ & -0.105 & -0.059 & 0.248 *** \\
\hline & & $\mathrm{G}$ & & & & & 1.000 & 0.061 & 0.139 & 0.101 & 0.036 & -0.118 & $0.160^{*}$ & 0.012 & $0.301 * *$ & -0.114 & -0.060 & $0.252 * *$ \\
\hline \multirow[t]{2}{*}{6} & \multirow{2}{*}{$\begin{array}{l}\text { Number of primary } \\
\text { branches/ plant }\end{array}$} & $\mathrm{P}$ & & & & & & 1.000 & $\begin{array}{l}-0.100 \\
\end{array}$ & -0.132 & 0.070 & $0.157^{*}$ & $0.206^{* * *}$ & $0.255^{* * *}$ & $0.221^{* *}$ & -0.127 & $\begin{array}{l}-0.029 \\
\end{array}$ & 0.133 \\
\hline & & $\mathrm{G}$ & & & & & & 1.000 & -0.129 & $-0.153^{*}$ & 0.095 & $0.189 *$ & $0.217 * *$ & $0.259 * *$ & $0.226^{* *}$ & -0.141 & -0.032 & 0.135 \\
\hline \multirow[t]{2}{*}{7} & \multirow{2}{*}{$\begin{array}{l}\text { Number of pods/ } \\
\text { plant }\end{array}$} & $\mathrm{P}$ & & & & & & & 1.000 & $0.722 * *$ & $0.352 * *$ & -0.064 & 0.012 & $-0.149 *$ & $0.254 * *$ & $0.190^{*}$ & 0.059 & $0.419 * *$ \\
\hline & & $\mathrm{G}$ & & & & & & & 1.000 & $0.906^{* * *}$ & $0.359 * *$ & $\begin{array}{l}-0.101 \\
\end{array}$ & 0.007 & $-0.165^{*}$ & $0.278^{* *}$ & $0.201 * *$ & 0.063 & $0.453 * *$ \\
\hline \multirow[t]{2}{*}{8} & \multirow{2}{*}{$\begin{array}{l}\text { Number of clusters/ } \\
\text { plant }\end{array}$} & $\mathrm{P}$ & & & & & & & & 1.000 & $-0.374^{* *}$ & -0.136 & -0.000 & -0.105 & $0.169 *$ & 0.122 & 0.010 & $0.272 * *$ \\
\hline & & $\mathrm{G}$ & & & & & & & & 1.000 & $\begin{array}{ll}-0.068 \\
\end{array}$ & $-0.211^{* *}$ & -0.020 & -0.125 & $0.190^{*}$ & $0.158^{*}$ & 0.017 & $0.318^{* * *}$ \\
\hline \multirow[t]{2}{*}{9} & \multirow{2}{*}{$\begin{array}{l}\text { Number of pods/ } \\
\text { cluster }\end{array}$} & $\mathrm{P}$ & & & & & & & & & 1.000 & 0.086 & $\begin{array}{c}-0.001 \\
\end{array}$ & $\begin{array}{c}-0.041 \\
\end{array}$ & 0.108 & 0.096 & 0.058 & $0.189 *$ \\
\hline & & $\mathrm{G}$ & & & & & & & & & 1.000 & $0.219 * *$ & 0.030 & -0.084 & $0.237^{* *}$ & 0.132 & 0.100 & $0.373 * *$ \\
\hline \multirow[t]{2}{*}{10} & \multirow{2}{*}{ Pod length $(\mathrm{cm})$} & $\mathrm{P}$ & & & & & & & & & & 1.000 & $0.401 * *$ & $0.527^{* * *}$ & $0.314 * *$ & 0.020 & 0.109 & $0.319 * *$ \\
\hline & & $\mathrm{G}$ & & & & & & & & & & 1.000 & $0.366^{* *}$ & $0.602 * *$ & $0.353^{* *}$ & -0.005 & 0.127 & 0.351 *** \\
\hline \multirow[t]{2}{*}{11} & \multirow{2}{*}{$\begin{array}{l}\text { Number of Seeds/ } \\
\text { pod }\end{array}$} & $\mathrm{P}$ & & & & & & & & & & & 1.000 & $0.158^{*}$ & $0.369^{* *}$ & -0.070 & 0.038 & $0.325^{* *}$ \\
\hline & & $\mathrm{G}$ & & & & & & & & & & & 1.000 & $0.167^{*}$ & $0.386^{* *}$ & -0.091 & 0.040 & $0.343 * *$ \\
\hline \multirow[t]{2}{*}{12} & Tect urigh (e) & $P$ & & & & & & & & & & & & 1.000 & $0.397 * *$ & 0.049 & -0.045 & 0.410 ** \\
\hline & (E) & $\mathrm{G}$ & & & & & & & & & & & & 1.000 & $0.398^{* *}$ & 0.052 & -0.045 & 0.421 ** \\
\hline 13 & Biological yield/ & $P$ & & & & & & & & & & & & & 1.000 & $-0.375^{* * *}$ & -0.045 & 0.718 ** \\
\hline & plant (g) & $\mathrm{G}$ & & & & & & & & & & & & & 1.000 & $-0.396^{* *}$ & -0.045 & 0.739 ** \\
\hline 14 & Horentindor & $\mathrm{P}$ & & & & & & & & & & & & & & 1.000 & 0.074 & 0.340 *** \\
\hline & 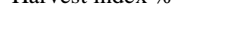 & $\mathrm{G}$ & & & & & & & & & & & & & & 1.000 & 0.077 & $0.307 * *$ \\
\hline 15 & Seed protein content & $\mathrm{G}$ & & & & & & & & & & & & & & & 1.000 & 0.006 \\
\hline & $\%$ & $P$ & & & & & & & & & & & & & & & 1.000 & 0.005 \\
\hline
\end{tabular}


Table.2 Genotypic path matrix for seed yield

\begin{tabular}{|c|c|c|c|c|c|c|c|c|c|c|c|c|c|c|c|c|c|}
\hline $\begin{array}{l}\mathbf{N} \\
\mathbf{0}\end{array}$ & Character & \begin{tabular}{|c|} 
Days to \\
$50 \%$ \\
flowerin \\
g
\end{tabular} & $\begin{array}{l}\text { Numbe } \\
\mathbf{r} \text { of } \\
\text { Flowers } \\
\text { / plant }\end{array}$ & \begin{tabular}{|c|} 
Numbe \\
$\mathbf{r}$ of \\
flowers \\
$/$ \\
cluster
\end{tabular} & \begin{tabular}{|c|} 
Days to \\
maturit \\
$y$
\end{tabular} & \begin{tabular}{|c|} 
Plant \\
height \\
(cm)
\end{tabular} & $\begin{array}{c}\text { Number } \\
\text { of } \\
\text { primary } \\
\text { branches } \\
\text { / plant }\end{array}$ & \begin{tabular}{|c|}
$\begin{array}{c}\text { Numbe } \\
\text { r of } \\
\text { pods/ } \\
\text { plant }\end{array}$ \\
\end{tabular} & \begin{tabular}{|l} 
Numbe \\
$\mathbf{r}$ of \\
clusters \\
/ plant
\end{tabular} & \begin{tabular}{|c|} 
Numbe \\
$\mathbf{r}$ of \\
pods/ \\
cluster
\end{tabular} & $\begin{array}{c}\text { Pod } \\
\text { length } \\
(\mathrm{cm})\end{array}$ & \begin{tabular}{|c|} 
Numbe \\
r of \\
seeds/ \\
pod
\end{tabular} & $\begin{array}{c}\text { Test } \\
\text { weight } \\
\text { (g) }\end{array}$ & $\begin{array}{c}\text { Biologica } \\
\text { l yield/ } \\
\text { plant (g) }\end{array}$ & $\begin{array}{c}\text { Harves } \\
t \text { index } \\
\%\end{array}$ & \begin{tabular}{|c|} 
Seed \\
protei \\
$n$ \\
conten \\
t \%
\end{tabular} & $\begin{array}{c}\text { Seed } \\
\text { yield/ } \\
\text { plant } \\
(\text { g. })\end{array}$ \\
\hline 1 & Days to $50 \%$ flowering & 0.078 & -0.006 & -0.019 & 0.061 & 0.006 & -0.008 & 0.004 & 0.011 & -0.014 & $\overline{-}$ & -0.039 & $\begin{array}{c}- \\
0.008\end{array}$ & -0.015 & 0.001 & $\begin{array}{c}- \\
0.007\end{array}$ & $-0.180 *$ \\
\hline 2 & Number of flowers/ plant & 0.010 & -0.129 & -0.011 & 0.014 & $\overline{-}$ & 0.008 & -0.120 & -0.102 & -0.064 & 0.011 & -0.006 & 0.017 & -0.041 & $\begin{array}{c}- \\
0.015\end{array}$ & $\overline{-}$ & $\begin{array}{c}0.429 * \\
*\end{array}$ \\
\hline 3 & Number of flowers/ cluster & -0.012 & 0.004 & 0.048 & -0.003 & 0.000 & 0.010 & -0.012 & -0.029 & 0.040 & 0.012 & 0.003 & 0.002 & 0.005 & 0.006 & $\begin{array}{c}- \\
0.001\end{array}$ & 0.019 \\
\hline 4 & Days to maturity & -0.073 & 0.010 & 0.007 & -0.093 & $\overline{-}$ & -0.013 & 0.006 & 0.003 & 0.008 & 0.022 & 0.025 & $\begin{array}{c}- \\
0.006\end{array}$ & -0.010 & 0.022 & 0.018 & -0.074 \\
\hline 5 & Plant height $(\mathrm{cm})$ & 0.000 & 0.001 & 0.000 & 0.001 & 0.005 & 0.000 & 0.001 & 0.001 & 0.000 & $\begin{array}{c}- \\
0.001\end{array}$ & 0.001 & 0.000 & 0.001 & 0.001 & 0.000 & $\begin{array}{c}0.252 * \\
*\end{array}$ \\
\hline 6 & $\begin{array}{l}\text { Number of primary branches/ } \\
\text { plant }\end{array}$ & -0.003 & -0.002 & 0.006 & 0.005 & 0.002 & 0.032 & -0.004 & -0.005 & 0.003 & 0.006 & 0.007 & 0.008 & 0.007 & 0.005 & $\begin{array}{c}- \\
0.001\end{array}$ & 0.135 \\
\hline 7 & Number of pods/ plant & 0.031 & 0.620 & -0.163 & -0.041 & 0.092 & -0.086 & 0.665 & 0.602 & 0.239 & $\begin{array}{c}- \\
0.067\end{array}$ & 0.005 & $\overline{-}$ & 0.185 & 0.134 & 0.042 & $\begin{array}{c}0.453 * \\
*\end{array}$ \\
\hline 8 & Number of clusters/ plant & -0.064 & -0.373 & 0.284 & 0.014 & $\begin{array}{c}- \\
0.048 \\
\end{array}$ & 0.072 & -0.428 & -0.472 & 0.032 & 0.100 & 0.010 & 0.059 & -0.090 & $\begin{array}{c}- \\
0.075 \\
\end{array}$ & $\begin{array}{c}- \\
0.008\end{array}$ & $\begin{array}{c}0.318 * \\
*\end{array}$ \\
\hline 9 & Number of pods/ cluster & 0.033 & -0.093 & -0.154 & 0.017 & $\begin{array}{c}- \\
0.007 \\
\end{array}$ & -0.018 & -0.067 & 0.013 & -0.188 & $\begin{array}{c}- \\
0.041 \\
\end{array}$ & -0.006 & 0.016 & -0.045 & $\begin{array}{c}- \\
0.025 \\
\end{array}$ & $\begin{array}{c} \\
0.019 \\
\end{array}$ & $\begin{array}{c}0.373 * \\
*\end{array}$ \\
\hline 10 & Pod length (cm) & 0.012 & 0.002 & -0.007 & 0.007 & 0.003 & -0.005 & 0.003 & 0.006 & -0.006 & $\begin{array}{c}- \\
0.028 \\
\end{array}$ & -0.010 & $\begin{array}{c}- \\
0.017\end{array}$ & -0.010 & 0.000 & $\begin{array}{c}- \\
0.004 \\
\end{array}$ & $\begin{array}{c}0.351 * \\
*\end{array}$ \\
\hline 11 & Number of seeds/ pod & -0.014 & 0.001 & 0.002 & -0.008 & 0.004 & 0.006 & 0.000 & -0.001 & 0.001 & 0.010 & 0.028 & 0.005 & 0.011 & $\begin{array}{c}- \\
0.003 \\
\end{array}$ & 0.001 & $\begin{array}{c}0.343 * \\
*\end{array}$ \\
\hline 12 & Test weight (g) & -0.003 & -0.003 & 0.001 & 0.002 & 0.000 & 0.006 & -0.004 & -0.003 & -0.002 & 0.014 & 0.004 & 0.023 & 0.009 & 0.001 & $\begin{array}{c}- \\
0.001 \\
\end{array}$ & $\begin{array}{c}0.421 * \\
*\end{array}$ \\
\hline 13 & Biological yield/ plant (g) & -0.188 & 0.320 & 0.107 & 0.103 & 0.210 & 0.225 & 0.276 & 0.189 & 0.236 & 0.352 & 0.384 & 0.396 & 0.995 & $\begin{array}{c}- \\
0.394 \\
\end{array}$ & $\begin{array}{c}- \\
0.045 \\
\end{array}$ & $\begin{array}{c}0.739 * \\
*\end{array}$ \\
\hline 14 & Harvest index \% & 0.011 & 0.077 & -0.083 & -0.156 & $\begin{array}{c}- \\
0.077 \\
\end{array}$ & -0.095 & 0.135 & 0.106 & 0.089 & $\begin{array}{c}- \\
0.004 \\
\end{array}$ & -0.061 & 0.035 & -0.266 & 0.672 & 0.052 & $\begin{array}{c}0.307 * \\
*\end{array}$ \\
\hline 15 & Seed protein content \% & 0.002 & -0.001 & 0.001 & 0.004 & 0.001 & 0.001 & -0.001 & 0.000 & -0.002 & $\begin{array}{c}- \\
0.002 \\
\end{array}$ & -0.001 & 0.001 & 0.001 & $\begin{array}{c}- \\
0.001 \\
\end{array}$ & $\overline{-}$ & 0.005 \\
\hline
\end{tabular}

$(\mathrm{R}$ square $=0.9761$ and Residual effect $=0.1547)$ 
Table.3 Phenotypic path matrix for seed yield

\begin{tabular}{|c|c|c|c|c|c|c|c|c|c|c|c|c|c|c|c|c|c|}
\hline No & Character & $\begin{array}{l}\text { Days to } \\
50 \% \\
\text { flowering }\end{array}$ & $\begin{array}{c}\text { Number } \\
\text { of } \\
\text { flowers/ } \\
\text { plant }\end{array}$ & $\begin{array}{c}\text { Number } \\
\text { of } \\
\text { flowers/ } \\
\text { cluster }\end{array}$ & $\begin{array}{c}\text { Days to } \\
\text { maturity }\end{array}$ & $\begin{array}{c}\text { Plant } \\
\text { height } \\
(\mathbf{c m})\end{array}$ & $\begin{array}{c}\begin{array}{c}\text { Number } \\
\text { of } \\
\text { primary } \\
\text { branches/ } \\
\text { plant }\end{array} \\
\end{array}$ & $\begin{array}{l}\text { Numbe } \\
\mathbf{r} \text { of } \\
\text { pods/ } \\
\text { plant }\end{array}$ & $\begin{array}{c}\text { Number } \\
\text { of } \\
\text { clusters/ } \\
\text { plant }\end{array}$ & $\begin{array}{l}\text { Number } \\
\text { of pods/ } \\
\text { cluster }\end{array}$ & $\begin{array}{c}\text { Pod } \\
\text { length } \\
(\mathbf{c m})\end{array}$ & $\begin{array}{c}\text { Number } \\
\text { of seeds/ } \\
\text { pod }\end{array}$ & $\begin{array}{c}\text { Test } \\
\text { weigh } \\
t(g)\end{array}$ & $\begin{array}{l}\text { Biological } \\
\text { yield/ } \\
\text { plant }(\mathrm{g})\end{array}$ & $\begin{array}{l}\text { Harvest } \\
\text { index \% }\end{array}$ & $\begin{array}{c}\text { Seed } \\
\text { protein } \\
\text { content } \\
\%\end{array}$ & $\begin{array}{c}\text { Seed } \\
\text { yield/ } \\
\text { plant } \\
\text { (g.) }\end{array}$ \\
\hline 1 & Days to $50 \%$ flowering & 0.021 & -0.001 & -0.004 & 0.012 & 0.00 & -0.001 & 0.001 & 0.003 & -0.002 & -0.003 & -0.006 & -0.002 & -0.003 & 0.001 & -0.002 & -0.094 \\
\hline 2 & Number of flowers/ plant & 0.001 & $-\mathbf{0 . 0 3 8}$ & -0.005 & 0.003 & -0.005 & 0.001 & -0.032 & -0.025 & -0.010 & 0.003 & -0.002 & 0.005 & -0.011 & $\cdots \cdots \cdot-0.005$ & -0.001 & $0.383 * *$ \\
\hline 3 & $\begin{array}{l}\text { Number of flowers/ } \\
\text { cluster }\end{array}$ & -0.001 & 0.001 & 0.007 & -0.000 & 0.000 & 0.001 & -0.001 & -0.004 & 0.004 & 0.000 & 0.000 & 0.000 & 0.000 & -0.000 & 0.000 & 0.012 \\
\hline 4 & Days to maturity & -0.025 & 0.004 & 0.002 & -0.044 & -0.006 & -0.006 & 0.002 & 0.000 & 0.003 & 0.007 & 0.010 & -0.003 & -0.004 & 0.008 & 0.008 & -0.061 \\
\hline 5 & Plant height (cm) & 0.002 & 0.003 & -0.000 & 0.004 & 0.026 & 0.002 & 0.003 & 0.002 & 0.001 & -0.003 & 0.004 & 0.000 & 0.008 & -0.003 & -0.002 & $0.248 * *$ \\
\hline 6 & $\begin{array}{l}\text { Number of primary } \\
\text { branches/ plant }\end{array}$ & -0.001 & -0.001 & 0.003 & 0.003 & 0.001 & 0.019 & -0.002 & -0.003 & 0.001 & 0.003 & 0.004 & 0.005 & 0.004 & -0.002 & -0.001 & 0.133 \\
\hline 7 & Number of pods/ plant & 0.010 & 0.129 & -0.019 & -0.009 & 0.020 & -0.016 & 0.157 & 0.114 & 0.055 & -0.010 & 0.002 & -0.023 & 0.040 & 0.030 & 0.009 & $0.419 * *$ \\
\hline 8 & $\begin{array}{l}\text { Number of clusters/ } \\
\text { plant }\end{array}$ & -0.012 & -0.056 & 0.050 & 0.001 & -0.008 & 0.012 & -0.063 & -0.088 & 0.032 & 0.012 & 0.000 & 0.009 & -0.015 & -0.011 & -0.001 & $0.272 * *$ \\
\hline 9 & Number of pods/ cluster & 0.006 & -0.017 & -0.042 & 0.004 & -0.001 & -0.005 & -0.023 & 0.025 & -0.066 & -0.006 & 0.000 & 0.003 & -0.007 & -0.006 & -0.004 & $0.189 *$ \\
\hline 10 & Pod length (cm) & 0.000 & 0.000 & -0.000 & 0.000 & 0.000 & -0.000 & 0.000 & 0.000 & -0.000 & -0.002 & -0.001 & -0.001 & -0.001 & 0.000 & -0.000 & $0.319 * *$ \\
\hline 11 & Number of seeds/ pod & -0.002 & 0.000 & 0.000 & -0.002 & 0.001 & 0.001 & 0.000 & 0.000 & 0.000 & 0.003 & 0.007 & 0.001 & 0.003 & -0.001 & 0.000 & $0.325 * *$ \\
\hline 12 & Test weight (g) & 0.000 & 0.000 & 0.000 & 0.000 & 0.000 & 0.000 & 0.000 & 0.000 & 0.000 & 0.000 & 0.000 & 0.000 & 0.000 & 0.000 & 0.000 & 0.410 *** \\
\hline 13 & Biological yield/ plant (g) & -0.125 & 0.274 & 0.043 & 0.097 & 0.289 & 0.213 & 0.244 & 0.163 & 0.104 & 0.302 & 0.356 & 0.382 & 0.963 & -0.361 & -0.043 & $0.718 * *$ \\
\hline 14 & Harvest index \% & 0.031 & 0.084 & -0.020 & -0.132 & -0.072 & -0.088 & 0.131 & 0.084 & 0.066 & 0.014 & -0.048 & 0.034 & -0.259 & 0.690 & 0.051 & $0.340 * *$ \\
\hline 15 & Seed protein content \% & 0.001 & -0.000 & 0.000 & 0.002 & 0.001 & 0.000 & -0.001 & -0.000 & -0.001 & -0.001 & -0.000 & 0.001 & 0.001 & -0.001 & -0.010 & 0.006 \\
\hline
\end{tabular}

$(\mathrm{R}$ square $=0.9518$ and Residual effect $=0.2196$ 
Yield (0.995) followed by harvest index (0.672), number of pods per plant (0.665), whereas number of flowers per plant $(-0.129)$, days to maturity $(-0.093)$, pod length $(-0.028)$, seed protein content $(-0.018)$ were contributed negative direct effect on seed yield. The present findings are also with the similar trends of result reported by Singh et al., (1990), Kutty et al., (2003) and Diriba Shanko et al., (2014).

Number of pods per plant (0.620) followed by biological yield (0.320) and harvest index (0.077) exhibited considerable positive indirect effect on seed yield per plant via number of flowers per plant. Such similar results were also reported by Uguru, (1995) and Nakawuka and Adipala (1999). Number of pods per plant (0.602) followed by biological yield (0.189) and harvest index (0.106) exhibited considerable positive indirect effect on seed yield per plant via number of cluster per plant by Tyagi and Koranne (1988), Patil et al., (1989) and Altinbas and Sepetogly (1993). Biological yield per plant (0.396) followed by number of cluster per plant (0.059), harvest index (0.035) and number of flowers per plant (0.017) exhibited considerable positive indirect effect on seed yield per plant via test weight by Kalaiyarasi and Palanisamy (2001) and Anbumalarmathi et al., (2005). Biological yield per plant (0.352) followed by number of clusters per plant (0.100) and days to maturity (0.022) exhibited considerable positive indirect effect on seed yield per plant via pod length by Uguru (1995), Nakawuka and Adipala (1999) and Driba Shanko et al., (2014). Biological yield per plant (0.210) followed by number of pods per plant (0.092), days to $50 \%$ flowering (0.006) and number of seeds per pod (0.004) exhibited considerable positive indirect effect on seed yield per plant via plant height by Kutty et al., (2003), Venkatesan et al., (2003) and Anbumalarmathi et al., (2005). Number of clusters per plant (0.284) followed by biological yield per plant (0.107), days to maturity (0.007) and number of primary branches per plant (0.006) exhibited considerable positive indirect effect on seed yield per plant via number of flowers per cluster by Tyagi and Koranne (1988), Patil et al., (1989) and Altinbas and Sepetogly (1993). Biological yield per plant (0.276) followed by harvest index (0.135) and days to maturity (0.006) exhibited considerable positive indirect effect on seed yield per plant via number of pods per plant by Tyagi and Koranne (1988) and Altinbas and Sepetogly (1993). Number of pods per plant (0.239) followed by biological yield per plant $(0.236)$ and harvest index (0.089) exhibited considerable positive indirect effect on seed yield per plant via number of pods per cluster by Kutty et al., (2003) and Driba Shanko et $a l$. , (2014). Biological yield per plant (0.225) followed by number of cluster per plant (0.072), number of flowers per cluster (0.010), number of flowers per plant (0.008) and test weight (0.006) exhibited considerable positive indirect effect on seed yield per plant via number of primary branches per plant by Tyagi and Koranne (1988) and Altinbas and Sepetogly (1993) and Meena et al., (2015). Number of pods per plant $(0.185)$ followed by number of seeds per pod (0.011) and test weight (0.009) exhibited considerable positive indirect effect on seed yield per plant via biological yield by Uguru (1995) and Kutty et al., (2003). The component of residual effect of path analysis was 0.1547 low residual effect indicated that character for path analysis were adequate and appropriate.

The direct and indirect effect of fifteen dependent characters on seed yield per plant as independent character was obtained in path coefficient analysis using phenotypic correlation coefficient are presented in table 3 . Path coefficient analysis revealed that the maximum positive direct effect was observed 
for biological yield (0.963) followed by harvest index (0.690), number of pods per plant (0.157), plant height (0.026), days to $50 \%$ flowering (0.021), number of primary branches per plant (0.019), number of flowers per cluster (0.007), number of seeds per pod (0.007) on seed yield per plant by Singh et al., (1990) and Kutty et al., (2003). Biological yield per plant $(0.382)$ followed by harvest index (0.034) and number of cluster per plant (0.009) had considerable positive indirect effect on seed yield per plant via test weight by Kalaiyarasi and Palanisamy (2001) and Anbumalarmathi et al., (2005). Biological yield per plant $(0.356)$ followed by days to maturity (0.010) and number of primary branches per plant $(0.004)$ had considerable positive indirect effect on seed yield per plant via number of seeds per pod by Tyagi and Koranne (1988) and Altinbas and Sepetogly (1993). Biological yield per plant (0.302) followed by harvest index (0.014) and number of clusters per plant (0.012) had considerable positive indirect effect on seed yield per plant via pod length by Uguru (1995), Nakawuka and Adipala (1999) and Driba Shanko et al., (2014). Biological yield per plant (0.289) and number of pods per plant (0.020) had considerable positive indirect effect on seed yield per plant via plant height. These results are in accordance with the findings of Kutty et al., (2003), Venkatesan et al., (2003) and Anbumalarmathi et al., (2005). Biological yield per plant (0.274) and number of pods per plant (0.129) had considerable positive indirect effect on seed yield per plant via number of flowers per plant. Such similar results were also reported by Uguru (1995) and Nakawuka and Adipala (1999). Biological yield per plant (0.244) and harvest index (0.131) had considerable positive indirect effect on seed yield per plant via number of pods per plant by Tyagi and Koranne (1988); Patil et al., (1989) and Altinbas and Sepetogly (1993). Biological yield per plant $(0.163)$ and number of pods per plant (0.114) had considerable positive indirect effect on seed yield per plant via number of clusters per plant by Tyagi and Koranne (1988) and Patil et al., (1989). The component of residual effects of path analysis was 0.219 low residual effect indicated that character for path analysis were adequate and appropriate.

Significant and positive correlations were observed between growth characters as well as between growth characters and seed yield of cowpea, when the correlation coefficients were partitioned into direct and indirect effects. Highest positive direct effect on biological yield per plant (0.963) followed by harvest index (0.690) and number of pods per plant (0.157). While, high indirect effect on seed yield per plant was exhibited by test weight (0.381), number of seeds per pod (0.356), pod length (0.302) and number of flowers per plant (0.274) through biological yield per plant. It is concluded from the path analysis study that seed yield in cowpea can be improved by focusing on character biological yield per plant, harvest index, number of pods per plant and plant height.

\section{References}

Al-jibouri, H.A., Millar, P.A. and Robinson, H.F. 1958. Genotypic and environmental variance and covariance in upland cotton crosses of interspecific origin. Agron. J., 50: 633-637.

Altinbas, M. and Sepetoglu, H. 1993. A study to determine components affecting seed yield in cowpea [Vigna unguiculata (L.) Walp.]. Populasyonumdadone verimini etkileyen ogelerin berlirlenmes uzerinde bircalisma doga turk tarum veormancilik dergisi, 17(3): 775-784.

Anbumalarmathi, J., Sheeba, A. and Deepasankar, P. 2005. Genetic variability and interrelationship studies 
in cowpea [Vigna unguiculata (L.) Walp.]. Res. Crops, 6(3): 517-519.

Arthur, M.A. 2009. Moisture-dependent physical properties of Cowpea. Unpublished B.Sc. Thesis, Department of Agricultural and Environmental Engineering, Niger Delt University, Bayelsa State. $64 \mathrm{Pp}$.

Bizeti, H.S., deCarvalho, C.G.P., deSouza, J.R.P., Destro, D. 2004. Path Analysis under Multicollinearity in Soybean. Agron. J., 47(5): 669-676.

Chaudhary, R.R., Joshi, B.K. 2005. Correlation and Path Coefficient Analyses in Sugarcane. Nepal Agri. Res. J., 6: 24-28.

Diriba Shanko, Mebeasellasie Andargie, Habtamu Zelleke. 2014. Interrelationship and Path Coefficient Analysis of Some Growth and Yield Characteristics in Cowpea (Vigna unguiculata L. Walp) Genotypes J. Plant Sci., 2(2): 97-101.

Dewey, O.R. and Lu, K.H. 1959. A correlation and Path coefficient analysis of components of crested wheatgrass seed production. Agron. J., 57: 515-518.

Fana, S.B., Pasquet, R.S., Gepts, P. 2004. Genetic diversity in cowpea (Vigna unguiculata L. Walp) as revealed by RAPD markers. Genetic Res. Crop Evol., 51: 539-550.

Fikru, M. 2004. Genetic variability and interrelationship of agronomic traits affecting seed Yield in desi type Chickpea (Cicer arietinum L.). An M.Sc thesis submitted to the School of Graduate Studies, Addis Ababa University.

Fisher, R.A. 1954. Statistical methods for research words. 12th Ed. Biol. Monograph and mannuals, 5: 130-131.

Kalaiyarasi, R. and Palanisamy, G.A. 2001. A study on character association and path Analysis in F4 generation of cowpea
[Vigna unguiculata (L.) Walp.]. Legume Res., 24(1): 36-39.

Kaveris, B., Salimath, P.M., Ravikumar, R.L. 2007. Genetic Studies in Greengram and Association analysis. Karnataka J. Agri. Sci., 20(4): 843-844.

Kutty, C.N., Mili, R., Jaikumaran, V. 2003, Correlation and path analysis in vegetable cowpea. Indian J. Hort., 60: 257-261.

Leleji, O.I. 1981. The extent of hybrid vigour for yield and yield components in cowpea (Vigna unguiculata (L.) Walp) in the Savana region of Nigeria. Nigerian J. Agri. Sci., 3(2): 141-148.

Manggoel, W., Uguru, M.I., Ndam, O.N. and Dasbak, M.A. 2012. Genetic variability, Correlation and path coefficient analysis of some yield components of ten cowpea [Vigna unguiculata (L.) Walp.] Accessions. J. Plant Breed. Crop Sci., 4(5): 80-86.

Mebeaselassie, A., Pasquet, R.S., Gowda, B.S., Muluvi, G.M., Timko, M.P. 2011. Construction of a SSR-based genetic map and identification of QTL for domestication traits using recombinant inbred lines from a cross between wild and cultivated cowpea (Vigna unguiculata L. Walp.). Mol. Breed., 28: 413-420.

Meena, H.K., Ram Krishna, K. and Singh Bhuri. 2015. Character associations between seed Yield and its components traits in cowpea [Vigna unguiculata (L.) Walp.]. Indian J. Agri. Res., 49(6): 567570.

Nakawuka, C.K., Adipala, E. 1999. A Path coefficient analysis of some yield component Interactions in cowpea. African Crop Sci. J., 7: 327- 331.

Padi, F.K. 2003. Correlation and path analysis of yield and yield components in pigeon pea. Pak. J. Biol. Sci., 6(19): 16891694. 
Pasquet, R.S. 2001. Allozyme diversity of cultivated cowpea (Vigna unguiculata L. Walp). Theoretical Appl. Genetics, 101: 211-219.

Patil, S.J., Venugoipal, R., Goud, J.V. and Parameshwar, R. 1989. Correlation and path Coefficient analysis in cowpea. Karnataka J. Agri. Sci., 2(3): 170-175.

Singh, K.B., Geletu, B., and Malhotra, R.S. 1990. Association of some traits with seed yield in Chickpea collections. Euphytica, 49: 83-88.

Tyagi, P.C. and Koranne, K.D. 1988. Correlation and path coefficient analysis in cowpea [Vigna unguiculata (L.) Walp.]. Indian J. Agri. Sci., 58(1): 57.

Uguru, M.I. 1996. Correlation and PathCoefficient analysis of major yield components in Vegetable Cowpea [Vigna unguiculata (L.) Walp.]. Proc.14th HORTSON Conference, AgoIwoye, 1-4 April, 1996.

Vange, T. and Egbe Moses, O. 2009. Studies on Genetic Characteristics of Pigeon Pea Germplasm at Otobi, Benue State of Nigeria, World J. Agri. Sci., 5(6): 714-719.

Veeraswamy, R., P. Rangaswamy, A.K. Fazlullah Khau and Muhammed Shereef. 1973. Heterosis in Cajanus cajan (L.) Millsp. Madras Agri. J., 60: 1317-1319.

Venkatesan, M., Prakash, M. and Ganesan. J. 2003. Correlation and path analysis in cowpea [Vigna unguiculata (L.) Walp.]. Legume Res., 26(2): 105-108.

\section{How to cite this article:}

Mahesh Sharma, P. P. Sharma, B. Upadhyay, H. L. Bairwa and Meghawal D. R. 2017. Character Association and Path Analysis in Cowpea [Vigna unguiculata (L.) Walp] Germplasm Line. Int.J.Curr.Microbiol.App.Sci. 6(6): 786-795. doi: https://doi.org/10.20546/ijcmas.2017.606.092 